\section{Musterbrief zur Mitgabe an Patienten \\ Bei der Patientin, bei dem Patien- ten ...... \\ besteht der Verdacht auf \\ Zur weiteren Abklärung sind folgende 0-III-Auftragsleistun- gen erforderlich: \\ Wie Sie wissen, ist für allergolo- gische Leistungen in der seit dem 1.7.1999 gültigen Laborreform kein Punktekontingent dafür vor- gesehen. Bitte teilen Sie mit, wie im Rahmen Ihrer Kasse die kor- rekte Abklärung ohne Bonus- minderung erfolgen soll.}

Die Allergologin hofft, nicht gegen Windmühlen zu kämpfen: ,Auch wenn sich nicht sofort etwas ändern dürfte, wird durch diese Maßnahmen doch eine gewisse Unruhe in die Reihen der Kassen gebracht." So wurde sie bereits von einer übergeordneten Stelle der AOK in Augsburg gebeten, die Schreiben an die Kassen doch zu unterlassen und vor allem die Patienten nicht zu verunsichern. Ein detailliertes Antwortschreiben der Dermatologin klärt die Verantwortlichen der Dienststellen über die Problematik bei der Allergieund Umweltdiagnostik auf.

Würschnitzer-Hünig: „Ich würde mich nun freuen, wenn mehr Kollegen unserem Beispiel folgten und ähnliche Briefe an die Kassen schrieben. Bis jetzt scheint aber eher das Gegenteil der Fall zu sein. Mein allergologisches Labor hat mir beispielsweise vor kurzem mitgeteilt, daß IgE-Untersuchungen auf potentielle Nahrungsmittelallergene $a b$ sofort nur noch einmal im Monat befundet werden können, da mit Beginn der Laborreform zu wenig Einsendungen eingehen würden."

Die Mahnung der Kollegin sollte ernstgenommen werden: „Ich glaube, wir tun uns da nichts Gutes, wenn wir uns in vorauseilendem Gehorsam wegen ein paar hundert Mark den Ast absägen, auf dem wir sitzen. Angriff ist die beste Verteidigung!“

$b k$

Nach persönlichen Informationen von B. Würschnitzer-Hünig, Kempten.

\title{
ÄDA-Forderungen im TV: Allergiepatienten gehören in die Hand des Experten!
}

\author{
"Qualifizierte Behandlung - in der Allergologie weiterhin \\ Glücksache". Dieses brisanten Themas nahm sich das \\ TV-Magazin „fact" an. Tenor des Beitrags: Vielen Allergiepatienten \\ könnte ein langer Leidensweg erspart bleiben, wenn sie gleich \\ einen Allergologie-Spezialisten aufgesucht hätten.
}

Die Fakten dürften vielen Zuschauern neu gewesen sein: Allergien haben bei uns mittlerweile den Charakter einer Volkskrankheit, Diagnose und Behandlung kosten die Volkswirtschaft rund 10 Milliarden DM jährlich. Doch liegt die kompetente allergologische Versorgung der Betroffenen trotz dieser hohen Kosten im argen: Nur knapp 10 Prozent der Patienten wird qualifiziert untersucht und behandelt, zu viele nicht entsprechend weitergebildete Ärzte aus dem niedergelassenen Bereich wagen sich an Krankheitsbilder, deren Komplexität sie nicht durchschauen - so zitierte ein Fernsehsprecher die Angaben des Ärzteverbandes Deutscher Allergologen (ÄDA). Die provokante These: „Fehlendes Expertenwissen, das zu Fehldiagnosen und -therapien führt."

Die Beispiele dafür waren gut gewählt. $\mathrm{Zu}$ Wort kamen Patienten, bei denen fälschlicherweise eine Nahrungsmittelallergie diagnostiziert wurde und die sich im Zustand der Mangelernährung in der Deutschen Klinik für Diagnostik in Wiesbaden vorstellten. Und erst dort wurde die Fehldiagnose erkannt.

Ein Grund für diese allgemein zu beobachtende Fehlentwicklung ist sicherlich die Tatsache, daß sich in Zeiten knapper werdender Ressourcen im Gesundheitswesen jeder der Beteiligten seinen Anteil zu sichern sucht. Um so dringender wird die Forderung nach der Verabschiedung eines Qualitätssicherungspapiers, das bei den Verant- wortlichen allerdings seit geraumer Zeit in der Schublade liegt. Auch die Einrichtung eines Lehrstuhls für Allergologie sollte überdacht werden.

Vielleicht hilft nun der öffentliche Druck. Immerhin erhielt der Präsident des ÄDA, Dr. Josef Wenning, die Gelegenheit, seine Überlegungen einem breiten Publikum zu präsentieren auch auf die Gefahr hin, den Unmut der nicht-qualifizierten niedergelassenen Kollegen auf sich zu ziehen.

Wenning: „Ein besserer Patientenschutz läßt sich nur durch den gezielten Einsatz von Fachärzten erreichen. Doch gibt es auf dem Gebiet der Allergologie bislang keine Qualitätsrichtlinien. Das führt zu der paradoxen Situation, daß Hausärzte, die die Weiterbildung zum Allergologen gar nicht haben, alle gebräuchlichen Methoden der allergologischen Diagnostik und Therapie ungehindert und nach Belieben ausschöpfen und anwenden können.“

Eine Lösung dieses Dilemmas könnten klar definierte Zuständigkeiten bieten: Hausärzte erkennen zunächst Allergiepatienten als solche und überweisen dann aber für die weitergehende Untersuchung und Therapie unbedingt an einen allergologisch weitergebildeten Facharzt.

$\mathrm{Ob}$ die Botschaft angekommen ist, wird das Patientenverhalten in der $\mathrm{Zu}$ kunft zeigen. Und vielleicht reagiert dann auch die Politik.

ARD-Magazin fact vom 27. September 1999. 